\title{
Gate-tunable superconducting weak link and quantum point contact spectroscopy on a strontium titanate surface
}

\author{
Patrick Gallagher, Menyoung Lee, James R. Williams and David Goldhaber-Gordon
}

\begin{abstract}
Two-dimensional electron systems in gallium arsenide and graphene have enabled ground-breaking discoveries in condensed-matter physics, in part because they are easily modulated by voltages on nanopatterned gate electrodes. Electron systems at oxide interfaces hold a similarly large potential for fundamental studies of correlated electrons and novel device technologies ${ }^{1-3}$, but typically have carrier densities too large to control by conventional gating techniques. Here we present a quantum transport study of a superconducting strontium titanate (STO) interface, enabled by a combination of electrolyte ${ }^{4-7}$ and metal-oxide gating. Our structure consists of two superconducting STO banks flanking a nanoscale STO weak link, which is tunable at low temperatures from insulating to superconducting behaviour by a local metallic gate. At low gate voltages, our device behaves as a quantum point contact that exhibits a minimum conductance plateau of $e^{2} / h$ in zero applied magnetic field, half the expected value for spin-degenerate electrons, but consistent with predictions ${ }^{8-12}$ and experimental signatures ${ }^{13-18}$ of a magnetically ordered ground state. The quantum point contact mediates tunnelling between normal and superconducting regions, enabling lateral tunnelling spectroscopy of the local superconducting state. Our work provides a generic scheme for quantum transport studies of STO and other surface electron liquids.
\end{abstract}

Transition metal oxides exhibit electronic ground states not seen in conventional semiconductors ${ }^{2,3}$. For instance, the interface between lanthanum aluminate and strontium titanate ${ }^{19}-$ two non-magnetic band insulators-hosts superconductivity ${ }^{20}$ and magnetism ${ }^{13-18}$. Rashba spin-orbit coupling at conductive STO interfaces ${ }^{21}$ has led to predictions of unconventional superconducting states $^{10}$, and of helical wires and Majorana fermions in nanoscale STO channels ${ }^{11}$. Furthermore, nanoscale lateral control of carriers could allow fabrication of gate-tunable, single-material Josephson junctions and superconducting quantum interference devices, as well as mesoscopic devices. However, few experiments on nanostructures in STO systems exist: superconductivity has been demonstrated in submicron regions tunable with a global back gate ${ }^{22}$, and channels sketched by a nanoscale tip show superconducting features ${ }^{23}$. In this work, we demonstrate the first realization of an all-STO, gate-tunable superconducting weak link. We tune our device to the ballistic 1D limit and perform superconducting spectroscopy that agrees with BCS weak-coupling expectations and previous work ${ }^{24}$.

Undoped STO is a band insulator. Inducing a two-dimensional (2D) electron density near $10^{13} \mathrm{~cm}^{-2}$ creates a metal, and several times that density creates an optimally doped superconducting state $^{4}$. To modulate the density on this scale, we use the electric double-layer transistor technique ${ }^{4-7}$. A schematic of our sample is shown in Fig. 1a. A single crystal of $\mathrm{TiO}_{2}$-terminated (100) $\mathrm{SrTiO}_{3}$ is patterned with ohmic contacts and a narrow metal top gate separated from the STO surface by a $5 \mathrm{~nm}$ alumina layer (Fig. $1 \mathrm{~b}$ and Methods). The sample is covered in ionic liquid, which is polarized by an electrode (not shown) to induce a $2 \mathrm{D}$ electron layer at the exposed STO surface; the area below the top gate remains insulating, because the gate screens the ions. The polarized sample is cooled to base temperature in a dilution refrigerator, freezing the ionic liquid and thus fixing the electron density in the exposed STO regions. The voltage on the top gate is then modulated to control transmission across the device, and conductance measurements are carried out with standard lock-in techniques in a four-terminal geometry (see Supplementary Information for filtering details).

The data reported in this Letter originate from a single cooldown of an STO channel $2 \mu \mathrm{m}$ in width spanned by a top gate of length $50 \mathrm{~nm}$ (other devices with similar behaviour are described in the Supplementary Information). The electron density in the STO regions exposed to the liquid was fixed near $5 \times 10^{13} \mathrm{~cm}^{-2}$, corresponding to $T_{\mathrm{C}} \approx 300 \mathrm{mK}$ (Supplementary Information). At $14 \mathrm{mK}$ and top gate voltage $V_{\mathrm{TG}}=0$, the device is insulating for source-drain bias voltage $V_{\mathrm{SD}}$ up to $1 \mathrm{mV}$ (left inset, Fig. 1c). At intermediate $V_{\mathrm{TG}}$, the $I V$ curve resembles that of an opaque tunnel junction, in which current flows only above a threshold $V_{\mathrm{SD}}$ (middle inset, Fig. 1c). This threshold decreases with increasing $V_{\mathrm{TG}}$ until a non-zero conductance appears at $V_{\mathrm{SD}}=0$. At the highest applied voltages $V_{\mathrm{TG}} \approx 3 \mathrm{~V}$, the $I V$ curve resembles that of a superconductor with critical current $I_{C}$ (right inset, Fig. 1c). We observe a normal-state resistance $R_{\mathrm{N}}>1 \mathrm{k} \Omega$ and a non-zero resistance $\mathrm{d} V / \mathrm{d} I$ below $I_{C}$, similar to other weak links with high normal-state resistance ${ }^{25}$.

Current-biased differential resistance measurements reveal that $I_{\mathrm{C}}$ is a strong function of $V_{\mathrm{TG}}$ (Fig. 2a). $I_{\mathrm{C}}$ can first be resolved around $V_{\mathrm{TG}}=2.1 \mathrm{~V}$, and increases to over $100 \mathrm{nA}$ by $V_{\mathrm{TG}}=3.2 \mathrm{~V}\left(I_{\mathrm{C}}\right.$ is defined as the lowest current at which the device reaches its normal-state resistance $R_{\mathrm{N}}$ ). $V_{\mathrm{TG}}$ is limited to $3.5 \mathrm{~V}$ by the breakdown of the alumina dielectric; for all data shown, gate leakage is below experimental sensitivity. The product $I_{\mathrm{C}} R_{\mathrm{N}}$, which is a measure of the superconducting gap $\Delta$ in the banks surrounding the weak $\operatorname{link}^{26}$, saturates near $150 \mu \mathrm{V}$ whereas $I_{\mathrm{C}}$ steadily rises with increasing $V_{\mathrm{TG}}$ (lower panel, Fig. 2a). For a short superconductor-normal-superconductor (SNS) junction in the dirty limit, Kulik-Omelyanchuk theory (KO-1; ref. 26) predicts that $e I_{\mathrm{C}} R_{\mathrm{N}} / \Delta \sim 2$, giving $\Delta \sim 75 \mu \mathrm{eV}$ in our sample. As described 

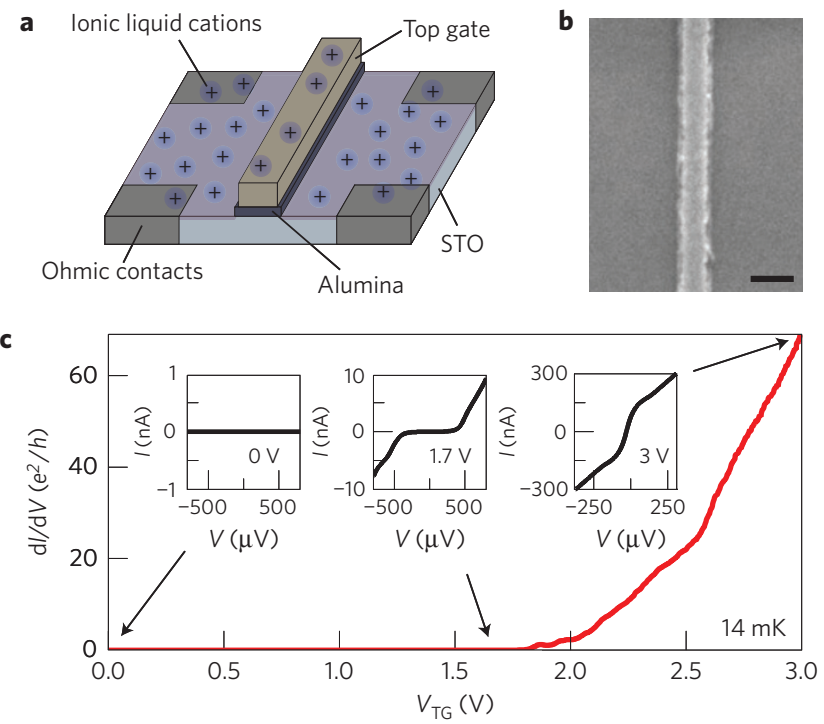

Figure 1 | Device properties. a, Schematic of the device, which is submerged in ionic liquid. Cations are drawn to the sample by a positively charged electrode (not shown) that is also immersed in the liquid, accumulating electrons at the exposed superconducting strontium titanate (STO) surface (lavender shading). The top gate defines a channel of low electron density by spacing and screening the cations. b, Scanning electron micrograph of a top gate of nominal length $60 \mathrm{~nm}$ (the device described in the text has length $50 \mathrm{~nm}$ ). The scale bar is $100 \mathrm{~nm}$. The alumina dielectric laterally protrudes 5-10 $\mathrm{nm}$ (faint edge around top gate). c, Electronic properties versus top gate voltage $V_{\mathrm{TG}}$ at $14 \mathrm{mK}$. Main panel: differential conductance at zero source-drain bias. Insets: IV curves from the insulating, tunnelling, and superconducting regimes.

below, we measure $\Delta \sim 80 \mu \mathrm{eV}$ by tunnelling spectroscopy, in agreement with KO-1. Furthermore, the saturating behaviour of $I_{\mathrm{C}} R_{\mathrm{N}}$ is expected: the top gate modulates the density in the weak link, which is largely independent of $\Delta$ in the banks. The above results are qualitatively insensitive to the exact definition of $I_{\mathrm{C}}$.

$I_{\mathrm{C}}$ decreases smoothly to zero in a magnetic field perpendicular to the plane (Fig. 2b). Were supercurrent flowing uniformly beneath the top gate, we would expect a Fraunhofer diffraction pattern ${ }^{27}$ for $I_{\mathrm{C}}(H)$, with zeros every $20 \mathrm{mT}$. Instead, the magnetic field response mirrors the diminishing superconductivity in the bulk. We suggest that only a small fraction of the area under our top gate becomes conductive, even at high $V_{\mathrm{TG}}$.

We next focus on the transition from tunnelling to superconducting behaviour. Figure 3a shows a waterfall plot of differential conductance versus source-drain voltage $V_{\mathrm{SD}}$ at intermediate $V_{\mathrm{TG}}$, measured in a voltage-biased configuration with $\mu_{0} H=0.25 \mathrm{~T}$ to suppress superconductivity. The traces cluster slightly below 1 and $2 e^{2} / h$ for zero bias, and near $1 / 2$ and $3 / 2 e^{2} / h$ for $V_{\mathrm{SD}} \approx 200 \mu \mathrm{V}$. We estimate a series resistance $\sim 2 \mathrm{k} \Omega \ll h / e^{2}$ (Supplementary Information), which would make the 1 and $2 e^{2} / h$ quantization quite accurate. This quantization appears as plateaux in $\mathrm{d} I / \mathrm{d} V$ as a function of $V_{\mathrm{TG}}$ for $V_{\mathrm{SD}}=0$ (Fig. 3d), and the plateau features form a crossing structure in $V_{\mathrm{SD}}-V_{\mathrm{TG}}$ space (Fig. $3 \mathrm{~b}$ ). These data strongly resemble measurements on ballistic quantum point contacts (QPCs) in clean 2D systems, where the zero-bias conductance is quantized to integer multiples of $G_{0}=g e^{2} / h$ for subband degeneracy $g$. The high-bias plateaux at half-integer multiples of $G_{0}$ are well documented ${ }^{28}$, and arise when the number of subbands below the Fermi level differs by one between the two leads.

The subbands seem to be non-degenerate $(g=1)$ even for $H=0$ (Fig. 3d). Two spin-degenerate QPCs connected in series without a

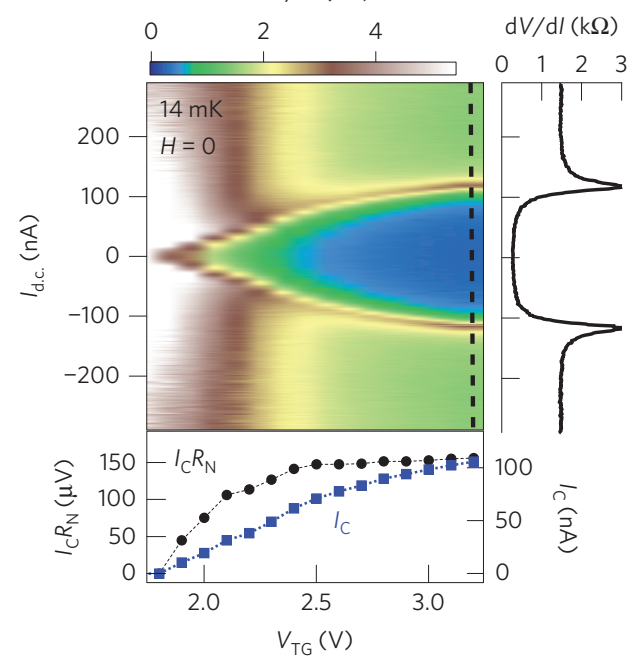

b

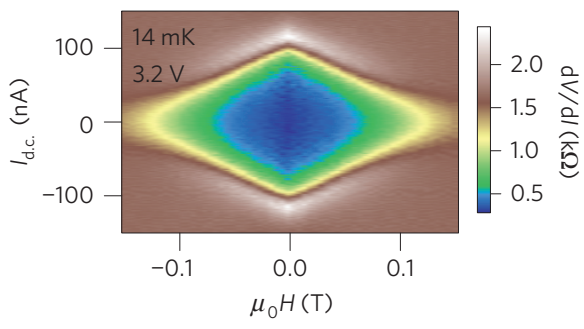

Figure 2 | Tunable superconducting weak link. a, Differential resistance versus top gate voltage and d.c. bias current $I_{\text {d.c. }}$. A critical current $I_{C}$ is clearly observed, and grows with increasing $V_{\mathrm{TG}}$. Right panel: $V_{\mathrm{TG}}=3.2 \mathrm{~V}$ (black dashed line in left panel). Lower panel: extracted $I_{C}$ and $I_{C} R_{N}$ product, where $R_{N}$ is the normal-state resistance measured at high $I_{\text {d.c. }}$. $I_{C} R_{N}$ saturates whereas $I_{C}$ continues to grow with increasing $V_{T G}$. b. Differential resistance versus applied perpendicular magnetic field and $I_{\text {d.c. }} \cdot I_{C}$ is suppressed with increasing magnetic field, but without a magnetic diffraction pattern.

phase coherence could mimic broken degeneracy in a single QPC, as their resistances $\left(h / 2 n e^{2}\right.$, integer $\left.n\right)$ would simply add. But this scenario would require nearly identical Fermi levels and dimensions for both QPCs, as we observe only one set of QPC features (Fig. 3b). The apparent broken degeneracy is more naturally explained by ferromagnetism, which has been observed ${ }^{13-18}$ in $\mathrm{LaAlO}_{3} / \mathrm{SrTiO}_{3}$ and is expected by theory: in some models, mobile $d$-electrons align their spins ${ }^{8,9}$, whereas in others itinerant electrons move in the Zeeman field of localized, spin-polarized $d_{x y}$ electrons $s^{10-12}$. Models that include a strong spin-orbit coupling ${ }^{21}$ alongside ferromagnetism in a 1D STO system predict a helical wire, again leading to an $e^{2} / h$ plateau $^{11}$; our data cannot distinguish between helical and spin-polarized pictures. Although we do not observe hysteresis, the coercive field could be below the resolution of our data $(\sim 5 \mathrm{mT})$, especially for small ferromagnetic patches ${ }^{9,16,17}$. Note that zero-field broken degeneracy in a QPC has also been reported in structures with an engineered in-plane electric field ${ }^{29}$, which is absent in our device.

The QPC structure at $H=0$ (Fig. 3c) remains largely unchanged from the $\mu_{0} H=0.25 \mathrm{~T}$ case, except for a slight splitting and sharpening of resonances. For $H=0$, however, two peaks emerge near zero bias between 1.9 and $2.05 \mathrm{~V}$, and evolve into one peak above $2.05 \mathrm{~V}$. We ascribe these peaks to a single superconductornormal (SN) interface; an SNS junction would give a large superconducting peak at zero bias ${ }^{27}$. For this gate voltage range, inelastic scattering probably transforms the junction into two 
a

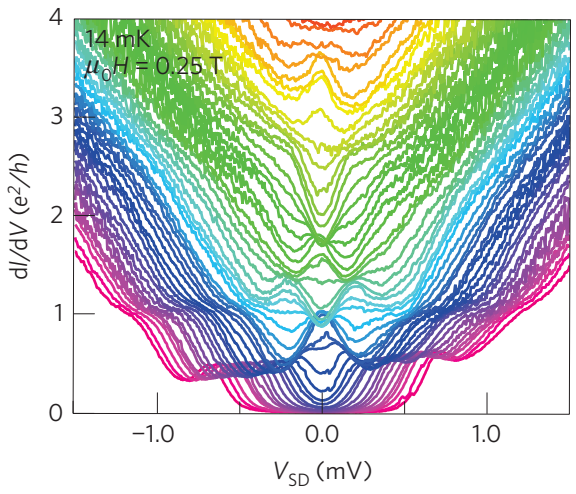

d
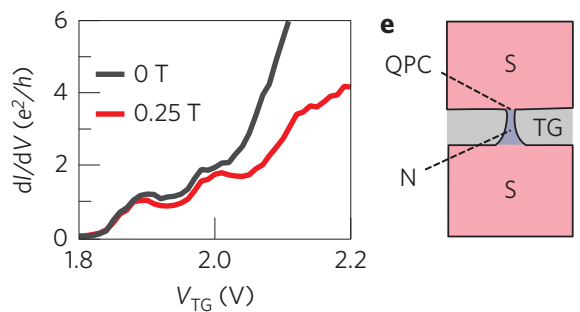

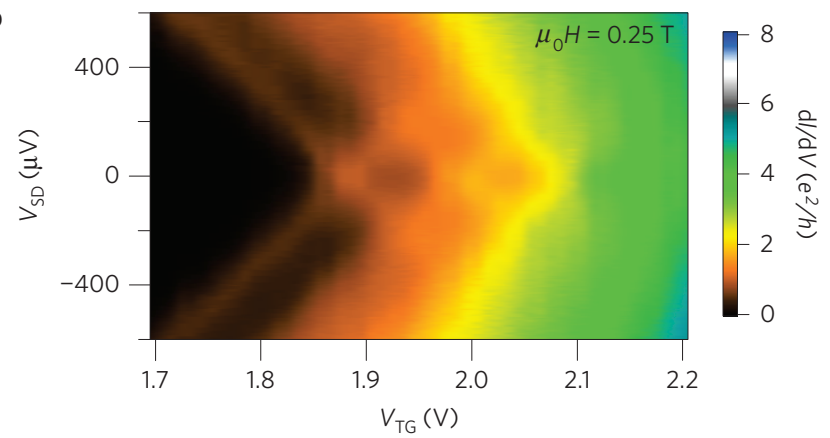

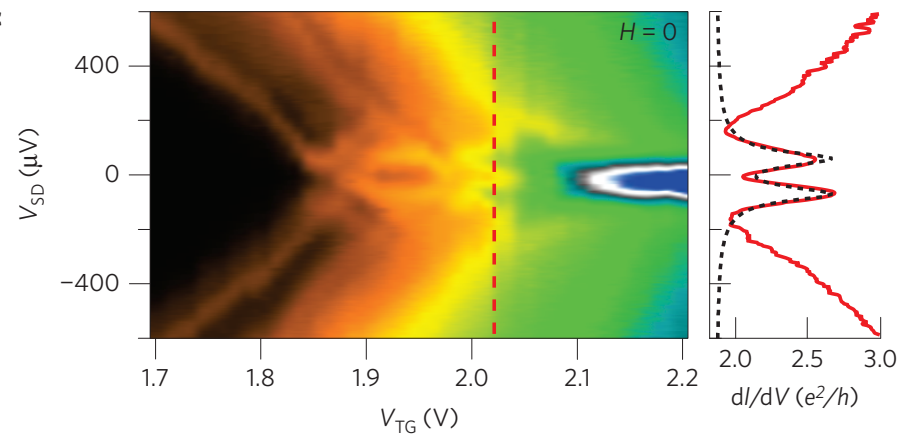

Figure 3 | Quantum point contact. a, Two-terminal differential conductance versus source-drain bias voltage $V_{S D}$ for top gate voltages from 1.76 V (violet) to $2.24 \mathrm{~V}$ (red), with no adjustments for contact resistance. A magnetic field of $0.25 \mathrm{~T}$ is applied to suppress superconductivity. The curves cluster near $e^{2} / h$ and $2 e^{2} / h$, as well as at the half-integers $e^{2} / 2 h$ and $3 e^{2} / 2 h$ for higher $V_{S D}$. $\mathbf{b}$, Same data as in a but the $V_{T G}$ range is offset by 60 m $V$ to correct for hysteresis and align features with $\mathbf{c}$. The crossing resonances track the subband minima. $\mathbf{c}$, Two-terminal $\mathrm{d} / / \mathrm{d} V$ as in $\mathbf{a}$ and $\mathbf{b}$ but with $H=0$ so that the banks are superconducting. Split peaks emerge around zero bias, merging into one strong superconducting peak at the highest $V_{\mathrm{TG}}$. Right panel:

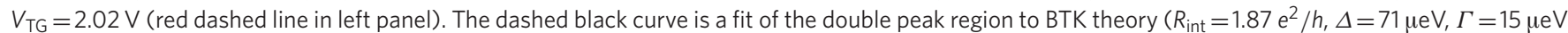

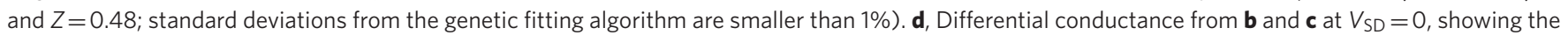
plateau structure. e, Cartoon of the electron distribution that gives rise to the quantum point contacts (QPC) behaviour. A normal channel (blue) beneath

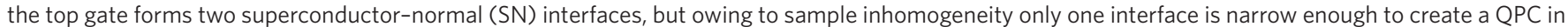
this gate voltage window. For $V_{\mathrm{TG}}<1.8 \mathrm{~V}$, the narrowest end of the channel completely closes, leading to a tunnelling $I V$ curve (Fig. $1 \mathrm{c}$ ); for $V_{\mathrm{TG}}>2.2 \mathrm{~V}$, the channel widens, yielding a superconducting IV curve.

SN interfaces in series, with current flow shaped by the higherresistance interface. The peaks are fitted well by the BlonderTinkham-Klapwijk (BTK) theory of SN interfaces,

$$
\frac{\mathrm{d} I}{\mathrm{~d} V}=\frac{1+Z^{2}}{R_{\mathrm{int}}} \int_{-\infty}^{\infty}-f^{\prime}(E-\mathrm{eV})(1+A(E ; \Delta, \Gamma, Z)-B(E ; \Delta, \Gamma, Z)) \mathrm{d} E
$$

where $f(E)$ is the Fermi function, $A(E)$ and $B(E)$ are probabilities of Andreev and normal reflection, and $\Gamma$ characterizes lifetime broadening $^{30}$. The interface barrier strength $Z$ accounts for elastic scattering, including reflections due to Fermi wavevector mismatch ${ }^{27} . R_{\text {int }}$ is the interface resistance in the absence of superconductivity, and equals the geometrical Sharvin resistance for $Z=0$. The right panel of Fig. $3 \mathrm{c}$ shows the high quality of the fit to equation (1) for a representative $V_{\mathrm{TG}}(2.02 \mathrm{~V}) . R_{\mathrm{int}}=0.53 \mathrm{~h} / \mathrm{e}^{2}$, which nearly agrees with the normal-state QPC resistance $0.58 \mathrm{~h} / \mathrm{e}^{2}$ at $V_{\mathrm{TG}}=2.02 \mathrm{~V}$. We thus conclude that the QPC itself is the $\mathrm{SN}$ interface that dominates the transport behaviour, as depicted in Fig. 3e.

The situation of a single-mode quantum wire in contact with a superconductor has been theoretically analysed ${ }^{31}$, and results in lineshapes nearly identical to BTK, justifying our use of equation (1). However, the small resonances that track the subband edges (moving diagonally in Fig. 3c) overlap the split peaks at some gate voltages-for example, $V_{\mathrm{TG}}=1.98 \mathrm{~V}$. To improve BTK fitting, we therefore divide the data of Fig. $3 \mathrm{c}$ by those of Fig. 3b. The resulting normalized $\mathrm{d} I / \mathrm{d} V$ traces and fits are shown in Fig. 4a. With increasing $V_{\mathrm{TG}}$, split peaks merge into a single zero-bias peak, which reaches the theoretical maximum conductance enhancement of 2 for an $\mathrm{SN}$ junction with zero barrier. As $V_{\mathrm{TG}}$ increases, the extracted $Z$ remains near 0.5 until the middle of the normal-state $2 e^{2} / h$ plateau $(2.03 \mathrm{~V})$, where $Z$ begins to drop to zero (Fig. $4 \mathrm{~b})$. The extracted $\Delta$ is $60-90 \mu \mathrm{eV}$, except near the plateau transitions $(1.97 \mathrm{~V}$ and $2.05 \mathrm{~V}$ ): there the normal-state conductance varies significantly at low bias, making both our normalization and our BTK model less accurate. Although statistical errors are small, systematic errors due to background features are significant (Supplementary Information). For $V_{\mathrm{TG}}>2.12 \mathrm{~V}$, the normalized conductance at zero bias grows larger than 2 and strong $\mathrm{d} I / \mathrm{d} V$ dips surround the central peak, signalling the transition to SNS behaviour.

A QPC with conductance $e^{2} / h$ is a half-metal, which typically gives zero Andreev reflection probability at an $s$-wave superconductor interface. But, in our sample, introducing superconductivity enhances the zero-bias conductance above $e^{2} / h$ (Fig. 3d), suggesting significant Andreev reflection. This conundrum may be resolved by spin-flip Andreev reflections resulting from spin-orbit coupling in the superconductor ${ }^{32}$, dephasing ${ }^{33}$, magnetization gradients ${ }^{34}$, and/or impurities ${ }^{35}$. Such processes would restore a BTK-like lineshape for split peaks on the $e^{2} / h$ plateau (Supplementary Information).

The BTK model yields spectroscopic information that agrees with our expectations for the superconducting state. $\Delta(T)$ extracted from BTK fits on the $2 e^{2} / h$ plateau (Fig. 4c) follows the BCS prediction $^{27}$ (Fig. $4 \mathrm{~d}$ ). $\Gamma$ grows slightly as $\Delta$ is suppressed to zero with increasing temperature, in agreement with tunnelling measurements on $\mathrm{LaAlO}_{3} / \mathrm{SrTiO}_{3}$ (ref. 24; the upturn in $\Gamma$ for 
a

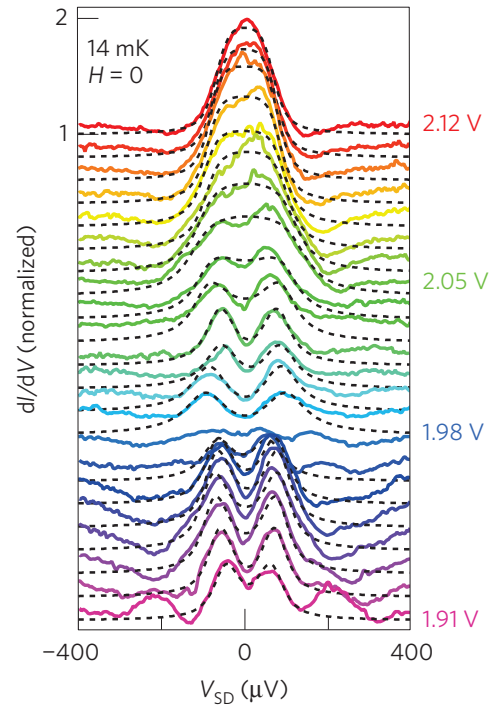

b

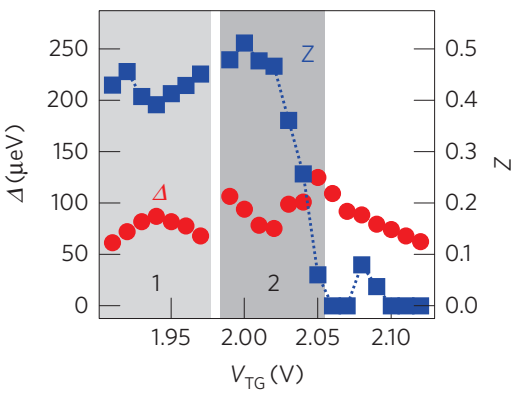

c

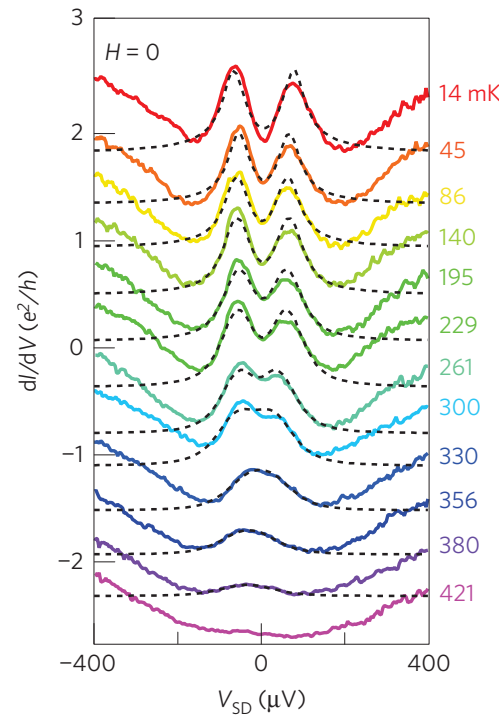

d

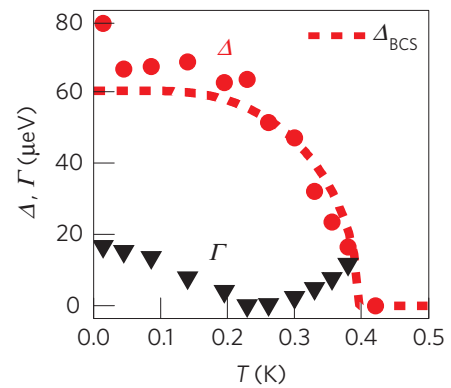

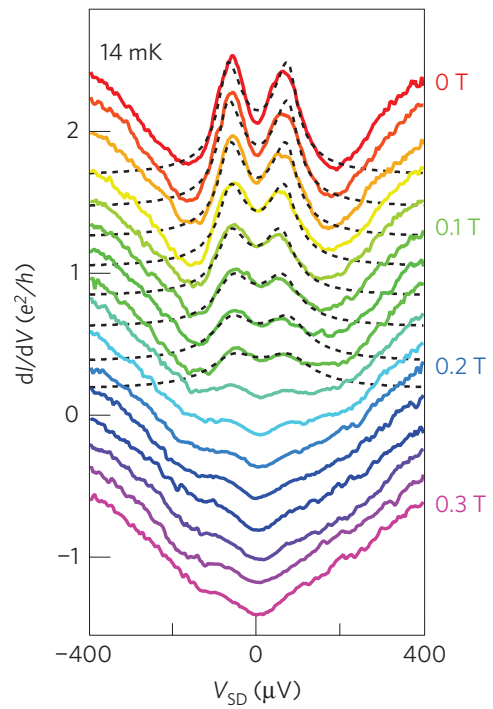

f

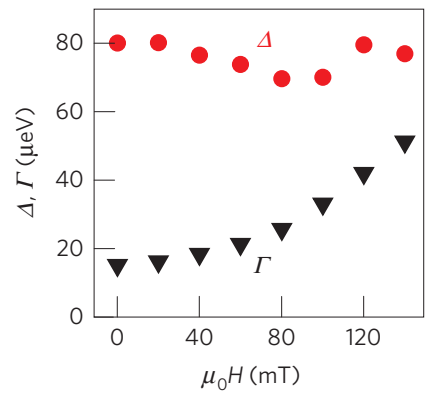

Figure 4 | Quantum point contact spectroscopy of the superconductor. a, Normalized $d / / d V$ for $1.91 \mathrm{~V}<V_{T G}<2.12 \mathrm{~V}$, calculated by dividing the data in Fig. $3 \mathrm{c}$ by the data in Fig. 3b. Dashed black curves are BTK fits. Traces for $V_{\mathrm{TG}}<2.12 \mathrm{~V}$ are successively offset downwards by 0.2 . b, Extracted barrier strength $Z$ (right axis) and gap $\Delta$ (left axis). The voltages corresponding to the 1 and $2 e^{2} / h$ plateaux at $\mu_{0} H=0.25 \mathrm{~T}$ are indicated with light and dark grey shading, respectively. The data at $1.98 \mathrm{~V}$ did not have well-defined peaks to fit. $\mathbf{c}$, Peaks at top gate voltage $V_{\text {TG }}=2.03 \mathrm{~V}$ for various temperatures, and BTK fits with $Z=0.5$ to the double peaks. Traces for $T>14 \mathrm{mK}$ are successively offset downwards by $0.4 \mathrm{e}^{2} / \mathrm{h}$. d, Extracted values of $\Delta$ and the broadening parameter $\Gamma$ versus temperature. Dashed red curve is a fit of $\Delta$ to weak-coupling BCS theory. e, Peaks at $V_{\mathrm{TG}}=2.08 \mathrm{~V}$ for various perpendicular magnetic fields, and BTK fits with $Z=0.4$. Traces for $H>0$ are successively offset downwards by $0.2 \mathrm{e}^{2} / \mathrm{h}$. Fits are not possible above $0.14 \mathrm{~T}$ owing to the increasingly relevant background features. The $V_{\mathrm{TG}}$ values quoted for $\mathbf{c}$ and $\mathbf{e}$ do not correspond exactly to $V_{\mathrm{TG}}$ in $\mathbf{a}$ owing to gate hysteresis on the order of $50 \mathrm{mV}$. f, Extracted values of $\Delta$ and $\Gamma$ versus magnetic field. Statistical error bars for all fits are smaller than the markers; systematic error is discussed in the Supplementary Information.

$T<200 \mathrm{mK}$ probably reflects elevated electron temperature, see Supplementary Information). In contrast, as the applied perpendicular magnetic field $H$ increases, $\Delta$ stays constant whereas $\Gamma$ increases markedly (Fig. 4e,f). This behaviour results from pair-breaking in a magnetic field and is observed in other superconductors such as $\mathrm{Nb}$ and $\mathrm{Sn}$ (ref. 36).

Our experiment illustrates the feasibility of clean transport measurements in nanostructured 2D oxide systems. Our demonstration of magnetism and tunable superconductivity in a single material with strong spin-orbit coupling lays the foundation for a host of interesting experiments. The gating technique presented here is generic, and can be implemented on other high-density surface electron systems.

\section{Methods}

Our samples are fabricated on $\mathrm{TiO}_{2}$-terminated (100) strontium titanate substrates (' $\mathrm{SrTiO}_{3}$ STEP substrates' from Shinkosha). Ohmic contacts and a large coplanar electrode are patterned by photolithography, followed by ion milling ${ }^{4}$ and evaporation of titanium and gold. The nanoscale gate is then defined in PMMA resist by $100 \mathrm{kV}$ electron-beam lithography. Following development and an ultraviolet ozone clean, $5 \mathrm{~nm}$ of alumina is grown via atomic layer deposition, a $5 \mathrm{~nm}$ titanium $/ 35 \mathrm{~nm}$ gold stack is deposited by evaporation, and the sample is immersed in acetone for liftoff. Finally, the mesa structure (Supplementary Information) is defined by a hard-baked photoresist mask.
Before measurement, a small drop of the ionic liquid 1-ethyl-3-methylimidazolium bis(trifluoromethanesulphonyl)amide (EMI-TFSI) is applied to the sample, which is then baked free of water in a tube furnace at $120^{\circ} \mathrm{C}$, under a $0.5 \mathrm{slm}$ flow of argon. The sample is loaded into the vacuum chamber of our dilution refrigerator, which is cryopumped by a surface at $77 \mathrm{~K}$. To minimize sample-degrading electrochemical effects, we cool the sample to $265 \mathrm{~K}$ before polarizing the liquid with $3.1 \mathrm{~V}$ on the coplanar electrode. We gradually cool the polarized sample to liquid helium temperatures over $36 \mathrm{~h}$, and then activate our dilution unit to reach $14 \mathrm{mK}$.

Received 6 January 2014; accepted 1 July 2014; published online 31 August 2014

\section{References}

1. Mannhart, J. \& Schlom, D. G. Oxide interfaces-An opportunity for electronics. Science 327, 1607-1611 (2010).

2. Zubko, P., Gariglio, S., Gabay, M., Ghosez, P. \& Triscone, J-M. Interface physics in complex oxide heterostructures. Annu. Rev. Condens. Matter Phys. 2, 141-165 (2011).

3. Hwang, H. Y. et al. Emergent phenomena at oxide interfaces. Nature Mater. 11, 103-113 (2012).

4. Ueno, K. et al. Electric-field-induced superconductivity in an insulator. Nature Mater. 7, 855-858 (2008).

5. Lee, Y. et al. Phase diagram of electrostatically doped $\mathrm{SrTiO}_{3}$. Phys. Rev. Lett. 106, 136809 (2011). 
6. Lee, M., Williams, J. R., Zhang, S., Frisbie, C. D. \& Goldhaber-Gordon, D. Electrolyte gate-controlled Kondo effect in $\mathrm{SrTiO}_{3}$. Phys. Rev. Lett. 107, 256601 (2011)

7. Li, M., Graf, T., Schladt, T. D., Jiang, X. \& Parkin, S. S. P. Role of percolation in the conductance of electrolyte-gated $\mathrm{SrTiO}_{3}$. Phys. Rev. Lett. 109, 196803 (2012)

8. Pentcheva, R. \& Pickett, W. Charge localization or itineracy at $\mathrm{LaAlO}_{3} / \mathrm{SrTiO}_{3}$ interfaces: Hole polarons, oxygen vacancies, and mobile electrons. Phys. Rev. B 74, 035112 (2006).

9. Pavlenko, N., Kopp, T., Tsymbal, E. Y., Sawatzky, G. A. \& Mannhart, J. Magnetic and superconducting phases at the $\mathrm{LaAlO}_{3} / \mathrm{SrTiO}_{3}$ interface: The role of interfacial Ti $3 d$ electrons. Phys. Rev. B 85, 020407 (2012).

10. Michaeli, K., Potter, A. C. \& Lee, P. A. Superconducting and ferromagnetic phases in $\mathrm{SrTiO}_{3} / \mathrm{LaAlO}_{3}$ oxide interface structures: Possibility of finite momentum pairing. Phys. Rev. Lett. 108, 117003 (2012).

11. Fidkowski, L., Jiang, H-C., Lutchyn, R. M. \& Nayak, C. Magnetic and superconducting ordering in one-dimensional nanostructures at the $\mathrm{LaAlO}_{3} / \mathrm{SrTiO}_{3}$ interface. Phys. Rev. B 87, 014436 (2013).

12. Banerjee, S., Erten, O. \& Randeria, M. Ferromagnetic exchange, spin-orbit coupling and spiral magnetism at the $\mathrm{LaAlO}_{3} / \mathrm{SrTiO}_{3}$ interface. Nature Phys. 9, 626-630 (2013)

13. Brinkman, A. et al. Magnetic effects at the interface between non-magnetic oxides. Nature Mater. 6, 493-496 (2007).

14. Dikin, D. A. et al. Coexistence of superconductivity and ferromagnetism in two dimensions. Phys. Rev. Lett. 107, 056802 (2011).

15. Ariando, et al. Electronic phase separation at the $\mathrm{LaAlO}_{3} / \mathrm{SrTiO}_{3}$ interface. Nature Commun. 2, 188 (2011).

16. Li, L., Richter, C., Mannhart, J. \& Ashoori, R. C. Coexistence of magnetic order and two-dimensional superconductivity at $\mathrm{LaAlO}_{3} / \mathrm{SrTiO}_{3}$ interfaces. Nature Phys. 7, 762-766 (2011).

17. Bert, J. A. et al. Direct imaging of the coexistence of ferromagnetism and superconductivity at the $\mathrm{LaAlO}_{3} / \mathrm{SrTiO}_{3}$ interface. Nature Phys. 7, 767-771 (2011)

18. Lee, J. S. et al. Titanium $d_{x y}$ ferromagnetism at the $\mathrm{LaAlO}_{3} / \mathrm{SrTiO}_{3}$ interface. Nature Mater. 12, 703-706 (2013).

19. Ohtomo, A. \& Hwang, H. Y. A high-mobility electron gas at the $\mathrm{LaAlO}_{3} / \mathrm{SrTiO}_{3}$ heterointerface. Nature 427, 423-426 (2004)

20. Reyren, N. et al. Superconducting interfaces between insulating oxides. Science 317, 1196-1199 (2007).

21. Caviglia, A. D. et al. Tunable Rashba spin-orbit interaction at oxide interfaces. Phys. Rev. Lett. 104, 126803 (2010).

22. Stornaiuolo, D. et al. In-plane electronic confinement in superconducting $\mathrm{LaAlO}_{3} / \mathrm{SrTiO}_{3}$ nanostructures. Appl. Phys. Lett. 101, 222601 (2012).

23. Cheng, G. et al. Anomalous transport in sketched nanostructures at the $\mathrm{LaAlO}_{3} / \mathrm{SrTiO}_{3}$ interface. Phys. Rev. X 3, 011021 (2013).

24. Richter, C. et al. Interface superconductor with gap behaviour like a high-temperature superconductor. Nature 502, 528-531 (2013).

25. Winkelmann, C. B., Roch, N., Wernsdorfer, W., Bouchiat, V. \& Balestro, F Superconductivity in a single- $\mathrm{C}_{60}$ transistor. Nature Phys. 5, 876-879 (2009).

26. Likharev, K. Superconducting weak links. Rev. Mod. Phys. 51, 101-159 (1979).
27. Tinkham, M. Introduction to Superconductivity 2nd edn (Dover, 2004).

28. Patel, N. et al. Evolution of half plateaus as a function of electric field in a ballistic quasi-one-dimensional constriction. Phys. Rev. B 44, 13549-13555 (1991).

29. Debray, P. et al. All-electric quantum point contact spin-polarizer. Nature Nanotech. 4, 759-764 (2009).

30. Pleceník, A., Grajcar, M., Beňačka, Š., Seidel, P. \& Pfuch, A. Finite-quasiparticle-lifetime effects in the differential conductance of $\mathrm{Bi}_{2} \mathrm{Sr}_{2} \mathrm{CaCu}_{2} \mathrm{O}_{y} / \mathrm{Au}$ junctions. Phys. Rev. B 49, 10016-10019 (1994).

31. Takagaki, Y. \& Ploog, K. Quantum point contact spectroscopy of $d$-wave superconductors. Phys. Rev. B 60, 9750-9754 (1999).

32. Duckheim, M. \& Brouwer, P. W. Andreev reflection from noncentrosymmetric superconductors and Majorana bound-state generation in half-metallic ferromagnets. Phys. Rev. B 83, 054513 (2011).

33. Béri, B. Dephasing-enabled triplet Andreev conductance. Phys. Rev. B 79, 245315 (2009)

34. Eschrig, M. \& Löfwander, T. Triplet supercurrents in clean and disordered half-metallic ferromagnets. Nature Phys. 4, 138-143 (2008).

35. Wilken, F. B. \& Brouwer, P. W. Impurity-assisted Andreev reflection at a spin-active half-metal-superconductor interface. Phys. Rev. B 85, 134531 (2012)

36. Naidyuk, Y., Häussler, R. \& Löhneysen, H. Magnetic field dependence of the Andreev reflection structure in the conductivity of SN point contacts. Physica B 218, 122-125 (1996).

\section{Acknowledgements}

We thank J-M. Triscone and J. Mannhart for helpful discussions concerning this work and S. Ilani for broader discussions concerning the properties of STO. Sample fabrication was supported by the Air Force Office of Science Research, Award No.

FA9550-12-1-02520. Sample measurement was supported by the MURI Program of the Army Research Office, Grant No. W911-NF-09-1-0398. Development of the ionic liquid gating technique was supported by the Center on Nanostructuring for Efficient Energy Conversion (CNEEC) at Stanford University, an Energy Frontier Research Center funded by the US Department of Energy, Office of Basic Energy Sciences under Award No. DE-SC0001060. P.G. acknowledges support from the DOE Office of Science Graduate Fellowship Program. M.L. acknowledges support from Samsung and Stanford University. J.R.W. and D.G-G. acknowledge support from the W. M. Keck Foundation.

\section{Author contributions}

P.G. designed the experiment, fabricated the sample, and performed the measurements. All authors contributed to data analysis. P.G. prepared the manuscript with input from all authors.

\section{Additional information}

Supplementary information is available in the online version of the paper. Reprints and permissions information is available online at www.nature.com/reprints.

Correspondence and requests for materials should be addressed to D.G-G.

\section{Competing financial interests}

The authors declare no competing financial interests. 